Bendi ng Loss Eval uat i ons of $\mathrm{Hol}$ ey $\mathrm{Fi}$ ber S Having a Core Consi st ing of an El I i pt i cal-Hol e Lat t i ce by Vari ous Approaches

\begin{tabular}{|l|l|}
\hline 著者 & EGUCH Nasashi, TSUJI Yasuhi de \\
\hline $\begin{array}{l}\text { j our nal or } \\
\text { publ i cat i on ti l l e }\end{array}$ & I EEE J our nal of Quant um El ect roni cs \\
\hline vol une & 46 \\
\hline number & 5 \\
\hline page $\mathrm{r}$ ange & $601-609$ \\
\hline year & $2010-02-25$ \\
\hline URL & ht t p: //hdl . handl e. net /10258/00010162 \\
\hline
\end{tabular}




\title{
Bending Loss Evaluations of Holey Fibers Having a Core Consisting of an Elliptical-Hole Lattice by Various Approaches
}

\author{
Masashi Eguchi, Member, IEEE and OSA, and Yasuhide Tsuji, Member, IEEE and OSA
}

\begin{abstract}
In general, an accurate evaluation of the bending loss of an optical fiber with an arbitrary refractive index profile is a cumbersome task, especially for holey fibers (HFs). In this paper, various approaches are applied and compared for the bending loss evaluations of single-polarization elliptical-hole core circular-hole HFs (EC-CHFs), which are circular-hole HFs having a core with an elliptical-hole lattice structure. Our simulation results show that when bending loss formulas for weakly guiding circular fibers are applied to a $\mathrm{HF}$ by regarding it as an approximate circular fiber having an equivalent step-index (SI) profile, the definition of the core region is the most crucial issue. Moreover, we also show that the results obtained by the approximate approach based on the bending loss formulas for the equivalent SI circular fiber are in good agreement with direct approaches with numerical simulations for the real EC-CHF structure, if the equivalent core radius is suitably defined for the real cross section.
\end{abstract}

Index Terms-Holey fiber, elliptical hole, single-polarization, bending loss, finite-element method, beam propagation method, EC-CHF.

\section{INTRODUCTION}

$\mathbf{P}$ OLARIZATION-STATE stabilization of two degenerate fundamental modes is required for the applications of optical fibers to coherent optical communication systems and fiber interferometers. Inducing an axially nonsymmetrical core profile is effective to stabilize the polarization states and various birefringent or single-polarization fibers using geometrical or stress effects have been proposed [1]-[3]. On the other hand, holey fibers (HFs) [4], [5] with high refractive-index contrast possess high controllability for their transmission characteristics [6] and an enormously high modal birefringence has recently been reported in a squeezed lattice elliptical-hole HF [7]. Single-polarization HFs have also been reported [8][10] and a novel single-polarization HF using the anisotropic fundamental-space filling mode (FSM) [11] of elliptical-hole lattices has recently been proposed [12], [13]. This HF is referred to as an elliptical-hole core circular-hole HF (EC-CHF), which is a circular-hole HF having a core with an ellipticalhole lattice structure, and possesses more peculiar transmission properties compared with conventional HFs. The radiation loss caused by a fiber bend is a crucial issue for a practical implementation. While, in general, an accurate evaluation of

M. Eguchi is with the Department of Photonics System Technology, Chitose Institute of Science and Technology, Chitose, 066-8655 Japan. Email: megu@ieee.org.

Y.Tsuji is with the Department of Electric and Electronic Engineering, Kitami Institute of Technology, Kitami, 090-8507 Japan. E-mail: tsujiya@mail.kitami-it.ac.jp. the bending loss of an optical fiber with an arbitrary refractive index profile is a cumbersome task, various approaches have been applied to bending loss calculations. Marcatili proposed an exact approach to derive the bending loss of a dielectric slab waveguide by solving the eigenvalue equation for a ring resonator [14]. However, it is difficult to apply this direct approach to the bending loss evaluation of a round fiber, and thus various approximate approaches have been devised. The easiest approach would be to use the bending loss formulas derived by Marcuse, for a slab waveguide [15], [16] and a round fiber [17], [18], and the formula [17] has been extended to a round fiber with a radially inhomogeneous profile by Sakai and Kimura [19]. Other typical approach with a numerical method uses an equivalent straight fiber model [20] based on a conformal transformation [21]. Moreover, recent dramatic progress in computer technology has made possible even the three dimensional (3D) direct numerical simulation of a curved waveguide using a beam propagation method (BPM) [22].

On the other hand, HFs have a complicated cross section without an axially symmetric profile and it makes the bending loss evaluation more difficult. Nevertheless, so far several papers have reported on the bending loss of HFs [11], [13], [23][26]. In this paper, we attempt to apply various approaches to the bending loss evaluation of a single-polarization ECCHF that has a significant mode field deviation from a typical mode field in standard fibers, and the applicability of the bending loss formula, which is derived for weakly guiding circular fibers, to such an EC-CHF with peculiar characteristics is demonstrated by a comparison with the accurately direct simulations for the real structure.

\section{Single-POLARIZATION ELLIPTICAL-HOLE CORE CIRCULAR-HOLE HOLEY FIBER}

An EC-CHF is a circular-hole HF having a core with an elliptical-hole lattice structure and shown in Fig. 1. When the effective-index of FSM (FSM index) in the cladding lattice is designed to lie between those of the slow and fast modes of the core lattice consisting of elliptical holes, the EC-CHF can guide only one polarization state [12], [13]. Figures 1(a) and 1(b) correspond to EC-CHFs having two mutually orthogonal directions of elliptical holes in a hexagonal arrangement of the core and are referred to as $y \mathrm{EC}$ - and $x \mathrm{EC}-\mathrm{CHFs}$, respectively [13]. We notice that the fundamental guided mode corresponds to the $\mathrm{HE}_{11}^{y}$ mode in the $y \mathrm{EC}-\mathrm{CHF}$ (Fig. 1(a)) and the $\mathrm{HE}_{11}^{x}$ mode in the $x$ EC-CHF (Fig. 1(b)), respectively. 
(a)

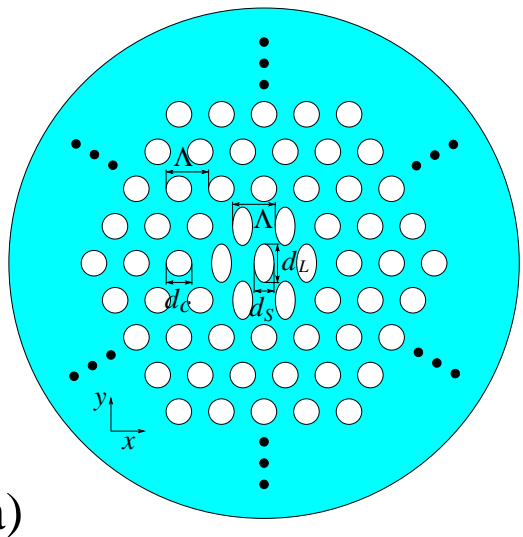

(b)

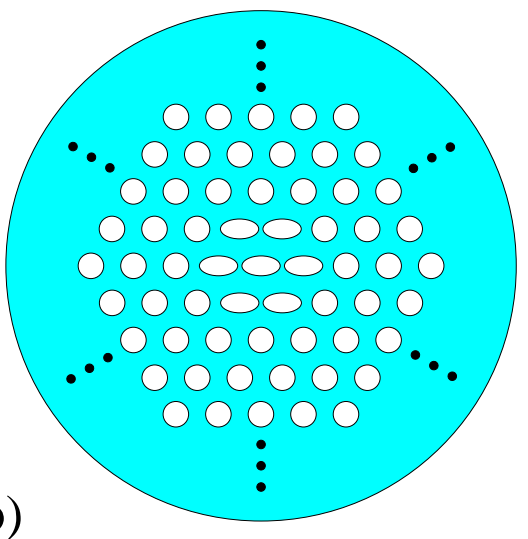

Fig. 1. Elliptical-hole core circular-hole HF. (a) The $y$ EC-CHF. (b) The $x$ EC-CHF.

\section{APPROACHES FOR BENDING LOSS EVALUATION OF HOLEY FIBERS}

In this section, we provide an overview of the approaches used for computing the bending loss of HFs. The 3D numerical simulation may be effective for an accurate evaluation of the bending losses of HFs, while such an accurate loss evaluation is a cumbersome task induced by huge computations, especially for arbitrary refractive index profiles including an airsilica periodic microstructure profile. Thus some approximations are usually introduced.

\section{A. Approximate approach based on bending loss formula} derived for a weakly guiding circular fiber

This easiest approximate approach applies an effectiveindex method to HFs [11], [13], [23], [25]. EC-CHFs consist of two kinds of air-hole lattices corresponding to a core and cladding regions. Each region is regarded as an infinite uniform lattice and is approximated by a medium having the refractive index equal to the FSM index of the air-hole lattice. Consequently, the EC-CHF can be regarded as an approximated circular fiber having an equivalent step-index (SI) profile, referred to as an equivalent SI fiber, and the following two bending loss formulas [17], [18] derived for

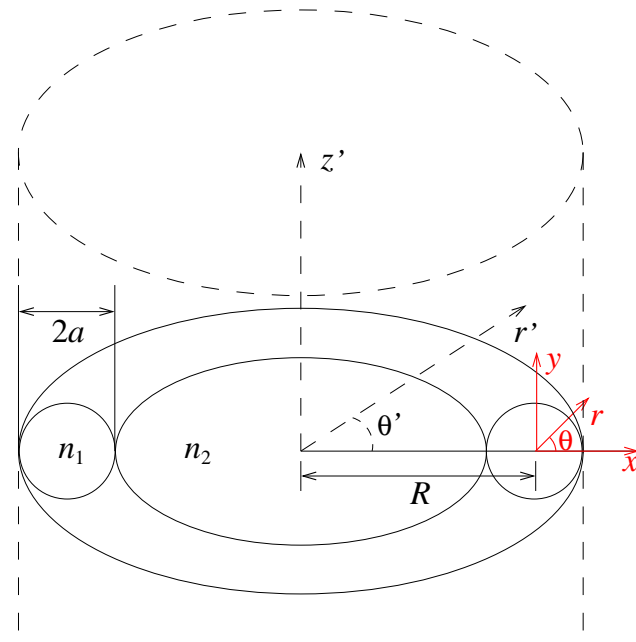

Fig. 2. Coordinate system for the standard loss formula (3).

a weakly guiding circular SI fiber are applicable to evaluate its bending loss.

1) Standard loss formula: Under the assumption that a fiber is bent uniformly with a constant radius $R$, as shown in Fig. 2, an outgoing wave from the fiber core is expressed as a superposition of cylindrical waves represented by an expansion of Hankel functions of the second kind, $H_{\nu}^{(2)}$, of the following form

$$
E_{z^{\prime} \nu}=B H_{\nu}^{(2)}\left(\kappa^{\prime} r^{\prime}\right) \exp \left(-i \nu \theta^{\prime}\right) \exp \left(-i \beta z^{\prime}\right)
$$

with

$$
\kappa^{\prime}=\left(n_{2}^{2} k_{0}^{2}-\beta^{2}\right)^{1 / 2},
$$

where $B$ is an expansion coefficient, $\beta$ is the phase constant for the $z^{\prime}$-direction, $n_{2}$ is the cladding index, and $k_{0}$ is the free-space wavenumber. Then the expansion coefficients are determined by matching the expanded field outside the fiber to the guided mode field on the fiber surface and the following standard loss formula [17] for an $\mathrm{LP}_{l m}$ mode in weakly guiding SI fibers can be derived:

$$
\alpha_{B}=\frac{\sqrt{\pi} \kappa^{2} \exp \left[-\frac{2}{3}\left(\gamma^{3} / \beta_{g}^{2}\right) R\right]}{2 \gamma^{3 / 2} v^{2} \sqrt{R} K_{l-1}(\gamma a) K_{l+1}(\gamma a)},
$$

where

$$
\begin{gathered}
\kappa=\left(n_{1}^{2} k_{0}^{2}-\beta_{g}^{2}\right)^{1 / 2}, \\
\gamma=\left(\beta_{g}^{2}-n_{2}^{2} k_{0}^{2}\right)^{1 / 2}, \\
v=k_{0} a\left(n_{1}^{2}-n_{2}^{2}\right)^{1 / 2} .
\end{gathered}
$$

$\beta_{g}$ is the propagation constant of the guided mode in the straight structure, $a$ is the core radius, $n_{1}$ is the core index, and $K_{l}(\gamma a)$ denotes the $l$ th-order modified Bessel function of the second kind. Using a numerical method for calculating the total power and the cladding field amplitude coefficient in the straight structure, we obtain the modified expression applicable to a fiber having an arbitrary profile core,

$$
\alpha_{B}=\sqrt{\frac{\varepsilon_{0}}{\mu_{0}}} \frac{\pi^{\frac{3}{2}} n_{\mathrm{eff}} A^{2}}{4 P_{g}} \frac{a \exp \left(\frac{-4 \Delta w^{3}}{3 a v^{2}} R\right)}{w\left(\frac{w R}{a}+\frac{v^{2}}{2 \Delta w}\right)^{1 / 2}},
$$




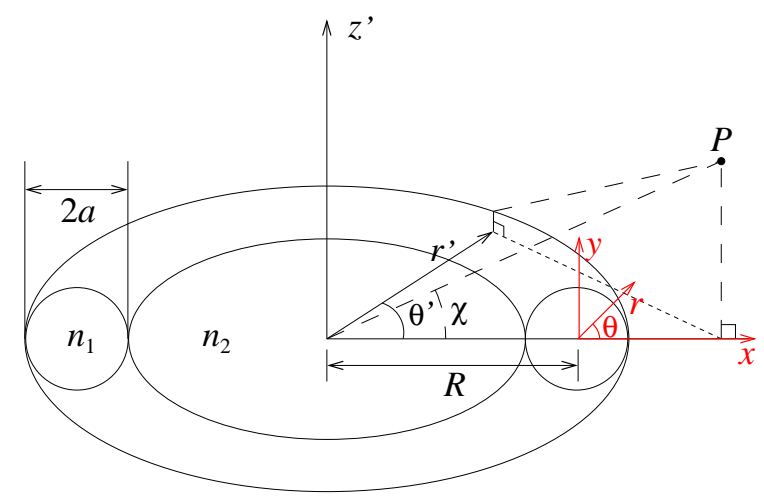

Fig. 3. Coordinate system for the formula (9) based on diffraction theory.

with

$$
P_{g}=\frac{1}{2} \int_{S} E_{x} H_{y}^{*}-E_{y} H_{x}^{*} d S,
$$

where $P_{g}$ and $A$ are the total power and the cladding field amplitude coefficient, respectively, in the straight fiber, $n_{\mathrm{eff}}=$ $\beta_{g} / k_{0}, \Delta=\left(n_{1}^{2}-n_{2}^{2}\right) /\left(2 n_{1}^{2}\right), w=a \gamma, \varepsilon_{0}$ and $\mu_{0}$ are the dielectric permittivity and magnetic permeability of free space, respectively, and the integral in (8) extends over the cross section. $x, y$, and $z$ ( $z$ is directed along the fiber axis) represent local Cartesian coordinates in the fiber cross section, as shown in Figs. 2 and 3. The validity of the formula (3) for standard HFs having a solid core, which is formed by a single air-hole defect region, has been demonstrated through a comparison with experimental results in [23].

2) Loss formula based on diffraction theory: Marcuse also proposed another approach based on Fraunhofer's diffraction theory to evaluate the bending losses of dielectric waveguides [18]. Under the scalar approximation, this loss formula is derived by using the scalar diffraction integral. In this approach, the field outside of the core is computed from the the guided mode field at the surface of the core with the threedimensional diffraction integral [16] and, by introducing some mathematical approximations that are required to calculate an integral, the following alternative loss formula for the coordinate system shown in Fig. 3 can be derived:

$$
\begin{aligned}
\alpha_{B} & =\sqrt{\frac{\varepsilon_{0}}{\mu_{0}}} \frac{n_{2} a^{2} A^{2}}{16 \pi P_{g}} \int_{-\pi / 2}^{\pi / 2} \frac{\exp \left[-\frac{2 R}{3 \beta_{g}^{2}}\left(\beta_{g}^{2}-n_{2}^{2} k_{0}^{2} \cos ^{2} \chi\right)^{3 / 2}\right]}{\sqrt{\beta_{g}^{2}-n_{2}^{2} k_{0}^{2} \cos ^{2} \chi}} \\
& \times F^{2}(\chi) \cos (\chi) d \chi
\end{aligned}
$$

with

$$
\begin{aligned}
F(\chi) & =-\gamma A \frac{K_{l-1}(w)+K_{l+1}(w)}{2} I_{1}+\left[n_{2} k_{0} \sin \chi I_{2}\right. \\
& \left.-\sqrt{\beta_{g}^{2}-n_{2}^{2} k_{0}^{2} \cos ^{2} \chi} I_{3}\right] A K_{l}(w),
\end{aligned}
$$

where $I_{1}, I_{2}$, and $I_{3}$ are defined in [18]. The above expression is modified to be applicable to fibers having an arbitrary profile core. For the dominant mode in weakly guiding SI fibers, which is denoted as $\mathrm{LP}_{01}$, (9) can be reduced to the formula shown in [18] with

$$
P_{g}=\frac{\pi v^{2} \beta_{g}|A|^{2}}{2 \omega \mu_{0} \gamma^{2}}\left|J_{l-1}(\kappa a) J_{l+1}(\kappa a)\right| .
$$

$J_{l}$ denotes the $l$ th-order Bessel function of the first kind.

In both the formulas (7) and (9), the total power $P_{g}$ can be expressed as follows:

for the guided mode in weakly guiding fibers,

$$
P_{g}=\frac{n_{\mathrm{eff}}}{2} \sqrt{\frac{\varepsilon_{0}}{\mu_{0}}} \int_{S} \Phi^{2} d S
$$

with

$$
\Phi=E_{x} \quad \text { or } \quad E_{y}
$$

and, for the $\mathrm{LP}_{01}$ mode in axially symmetric fibers,

$$
P_{g}=\frac{\pi \beta_{g}}{k_{0}} \sqrt{\frac{\varepsilon_{0}}{\mu_{0}}} P_{0}
$$

with

$$
P_{0}=\int_{0}^{\infty} \phi(r) r d r
$$

Here, we consider that the guided modal field $\Phi$ is expressed by

$$
\Phi=\phi(r)\left[\begin{array}{c}
\cos (l \theta) \\
\sin (l \theta)
\end{array}\right] \exp \left(-i \beta_{g} R \theta^{\prime}\right) .
$$

In general, in applying such formulas to HFs, it should be noted that since the core region can not be clearly defined, the accuracy of the obtained results strongly depends on the definition of core region. Moreover, since an uniform cladding is assumed in the formulas, the real cladding model of HFs consisting of a finite lattice cannot be included in this approximate approach. Accurate results may be obtained by using a two dimensional (2D) numerical analysis for calculating the total power, the cladding field amplitude coefficient, and the effective index in the formula, (7) or (9), for a real fiber cross section.

\section{B. Complex eigenmode analysis approach}

While a full 3D numerical simulation is quite effective for faithfully evaluating waveguides having a complicated cross section, such as HFs, it requires huge computations due to the discretization of the cross section. However, in bending loss evaluations, it is usually assumed that a fiber is bent uniformly with a constant radius. In this case, a bent fiber structure can be described in a cylindrical coordinate system or replaced by a straight fiber with an equivalent refractive index distribution, referred to as the equivalent straight structure model, and then the bending loss can be calculated directly from a complex eigenmode analysis for the cross section. Using a numerical method for a cross-sectional analysis, we can faithfully evaluate real structure models, including a cladding with a finite lattice structure.

1) Approach using a cylindrical coordinate system: From Maxwell's equation, we obtain the vector wave equation

$$
\nabla \times(p \nabla \times \boldsymbol{\Phi})-k_{0}^{2} q \boldsymbol{\Phi}=0,
$$

where $\boldsymbol{\Phi}$ represents either the electric field $\boldsymbol{E}$ or the magnetic field $\boldsymbol{H}$ and

$$
\begin{array}{cccc}
p=1, & q=n^{2} & \text { for } & \boldsymbol{\Phi}=\boldsymbol{E}, \\
p=1 / n^{2}, & q=1 & \text { for } & \boldsymbol{\Phi}=\boldsymbol{H} .
\end{array}
$$


For a bent fiber described in a cylindrical coordinate system shown in Fig. 2, the rotation operator is defined as

$$
\nabla \times \boldsymbol{\Phi}=\left[\begin{array}{ccc}
0 & -\frac{\partial}{\partial z} & \frac{1}{r} \frac{\partial}{\partial \theta} \\
\frac{\partial}{\partial z} & 0 & -\frac{\partial}{\partial r} \\
-\frac{1}{r} \frac{\partial}{\partial \theta} & \frac{1}{r}+\frac{\partial}{\partial r} & 0
\end{array}\right]\left[\begin{array}{c}
\Phi_{r} \\
\Phi_{\theta} \\
\Phi_{z}
\end{array}\right]
$$

When the distance along the fiber axis $z$ is approximated by $z \simeq R \theta^{\prime}$, the coordinates $r^{\prime}-R, z^{\prime}$, and $R \theta^{\prime}$ in a cylindrical coordinate system can be replaced by $x, y$, and $z$, respectively, in a Cartesian coordinate system, whose origin is placed at the center of the core cross section. Then, the rotation operator in the vector wave equation can be given by

$$
\nabla \times \boldsymbol{\Phi}=\left[\begin{array}{ccc}
0 & \frac{R}{R+x} \frac{\partial}{\partial z} & -\frac{\partial}{\partial y} \\
-\frac{R}{r+x} \frac{\partial}{\partial z} & 0 & \frac{1}{R+x}+\frac{\partial}{\partial x} \\
\frac{\partial}{\partial y} & -\frac{\partial}{\partial x} & 0
\end{array}\right]\left[\begin{array}{c}
\Phi_{x} \\
\Phi_{y} \\
\Phi_{z}
\end{array}\right]
$$

The approach using a cylindrical coordinate system is to solve the vector wave equation (17) with the help of this operator.

2) Approach using the equivalent straight structure model: Based on the conformal transformation [21], the equivalent refractive index distribution for the bent fiber is approximated by

$$
\begin{aligned}
n_{\mathrm{eq}}(x, y) & =n(x, y) \exp \left(\frac{x}{R}\right) \\
& \simeq n(x, y)\left(1+\frac{x}{R}\right),
\end{aligned}
$$

and then a vector wave equation can be expressed as

$$
\nabla \times\left(p_{\mathrm{eq}} \nabla \times \mathbf{\Phi}\right)-k_{0}^{2} q_{\mathrm{eq}} \mathbf{\Phi}=0
$$

where

$$
\begin{aligned}
& p_{\text {eq }}=1, \quad q_{\text {eq }}=n_{\text {eq }}^{2} \quad \text { for } \quad \boldsymbol{\Phi}=\boldsymbol{E}, \\
& p_{\text {eq }}=1 / n_{\text {eq }}^{2}, \quad q_{\text {eq }}=1 \quad \text { for } \quad \boldsymbol{\Phi}=\boldsymbol{H} \text {. }
\end{aligned}
$$

The approach using the equivalent straight structure model is to solve this vector wave equation.

In this paper, a 2D vector finite-element method (V-FEM) based on curvilinear edge/nodal hybrid elements is applied to the complex eigenmode analysis for (17) or (24), and the bending loss is obtained from the imaginary part of the obtained complex propagation constant.

\section{3D beam propagation method approach}

A 3D BPM approach [27] may be the most powerful and direct technique to accurately analyze real structure models including even a nonuniform bend. However, since HFs have a complicated cross-sectional structure, the accuracy is limited by computer resources. Assuming that the curvature is uniform, a cylindrical coordinate system or the equivalent straight structure model [28] is available, and thus it reduces the dimension of the discretization. In our BPM analysis, a cylindrical coordinate system is adopted.

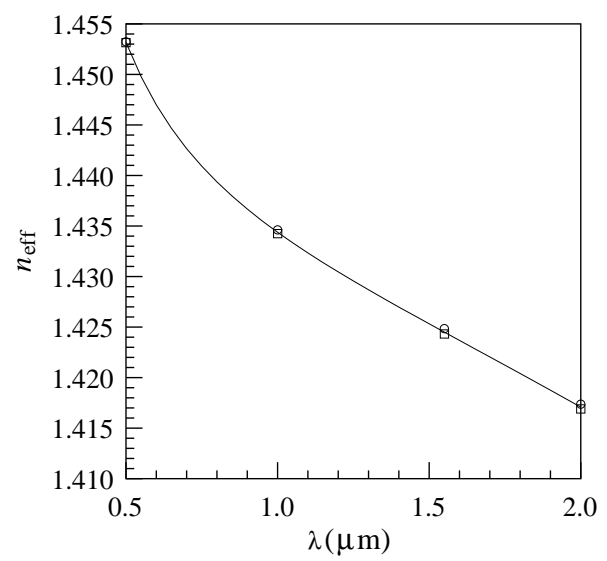

Fig. 4. Dispersion property for the guided mode of a single-polarization three-ring $y \mathrm{EC}-\mathrm{CHF}$ (solid curve) and equivalent SI fibers with $a=3.5 \Lambda$ (open circles) and $a=3 \Lambda$ (open squares).

\section{ASSESSMENT OF APPROACHES FOR EVALUATING BENDING LOSS}

We compute the bending loss of a $y \mathrm{EC}-\mathrm{CHF}$, as shown in Fig. 1(a), having 37 elliptical holes in the core (referred to as the three-ring core) by various approaches. The structure in Fig. 1 displays the one-ring EC-CHF, as an example of EC-CHFs. The EC-CHF evaluated here is characterized by a lattice pitch $\Lambda$, a circular hole size $\xi=d_{c} / \Lambda=0.23$, an elliptical hole size $\xi_{e}=d_{L} / \Lambda=0.4025$, and an ellipticity $d_{L} / d_{S}=3.6$. Here, $d_{L}$ and $d_{S}$ represent the major- and minoraxis lengths, respectively, and the refractive index is assumed to be 1.444 . Figure 4 shows the dispersion property of the three-ring $y$ EC-CHF with these parameters by the solid curve. In this figure, the effective indices of the equivalent SI fibers with core radii of $3.5 \Lambda$ and $3.0 \Lambda$ are also plotted by the open circles and the open squares, respectively. We observed good agreement among the three-ring $y \mathrm{EC}-\mathrm{CHF}$ and the equivalent SI fibers.

We first discuss the approximate approaches based on the loss formulas, in which the core index $n_{1}$ and cladding index $n_{2}$ are approximated by the FSM indices in the core and cladding regions, respectively, of the EC-CHF. A wavelength is hereafter assumed to be $1.55 \mu \mathrm{m}$. The bending loss obtained by the approach based on the standard loss formula (3) for the equivalent SI fiber, which we refer to as a formula approach based on the equivalent SI fiber, is indicated by the thin solid curves in Fig. 5, and, in addition, the thin dashed and thin dashed-dotted curves are obtained by using the standard loss formula (7) and the formula (9) based on diffraction theory, respectively, with a numerical method. A numerical method is not indispensable for SI circular fibers. However, here, to obtain the total power (14) and the amplitude coefficient $A$ in the formulas, we used the approximate scalar FEM for a circularly symmetric structure [29]-[31] as a numerical method and divided the core into quadratic ring elements [29]. The excellent agreement of the dashed and solid curves validates the approach using the standard loss formula (7) with the approximate scalar FEM. On the other hand, some discrepancies appear between the standard loss 


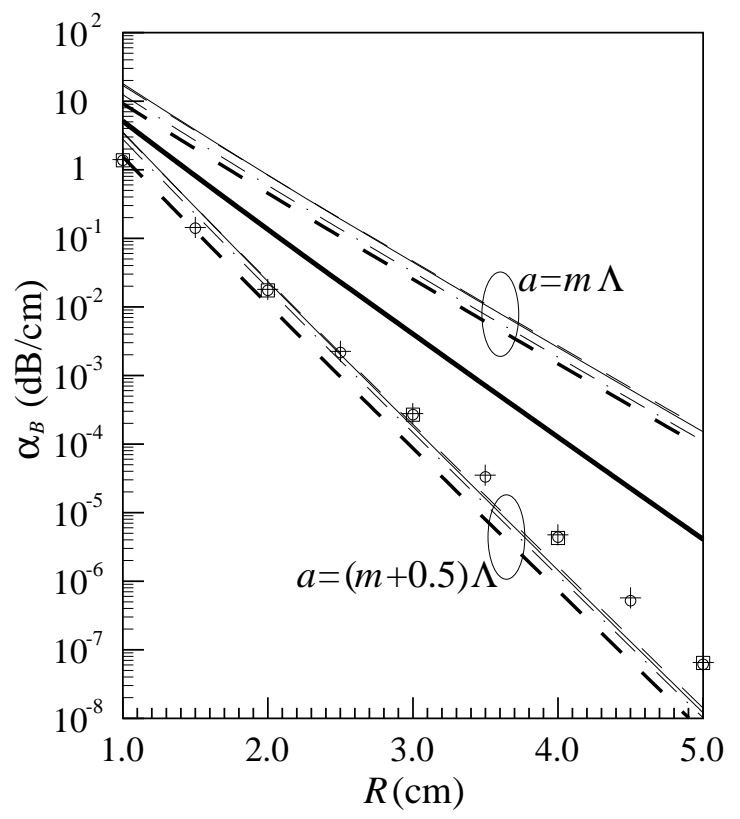

Fig. 5. Bending loss property of a three-ring $y \mathrm{EC}-\mathrm{CHF}$. Thin solid curves, the formula approach using (3) based on the equivalent SI fiber. Thin dashed curves, the formula approach using (7) based on the equivalent SI fiber with the approximate scalar FEM. Thin dashed-dotted curves, the formula approach using (9) based on the equivalent SI fiber with the approximate scalar FEM. Thick solid curves, the formula approach using (7) based on the equivalent SI fiber in which the real EC-CHF structure is considered for evaluating all the parameters in (7) $\left(P_{g}, A\right.$, and $\left.n_{\text {eff }}\right)$. Thick dashed curves, the formula approach using (7) based on the equivalent SI fiber in which the real ECCHF structure is considered only for evaluating $P_{g}$ and $A$. Open circles and pluses, the $2 \mathrm{D}$ complex eigenmode analyses using a cylindrical coordinate system and the equivalent straight structure model, respectively, for the real EC-CHF structure. Squares, the 3D V-FEBPM for the real EC-CHF structure.

formula (dashed or solid curve) and the formula based on diffraction theory (dashed-dotted curve), but the behaviors of bending losses with a bending radius $R$ agree well with each together. The dependence of an equivalent core radius, which is used as the core radius $a$ in the formulas, is also shown in Fig. 5. Here, a comparison of the results obtained for two equivalent core radii $a=m \Lambda$ and $(m+0.5) \Lambda$ is presented. $m$ represents the number of air-hole ring in the core lattice and here $m=3$. Noticeable discrepancies appear between both the results, and it should be noted that the definition of the equivalent core radius strongly affects the accuracy of bending loss evaluations using the effective-index approach based on the loss formula. Moreover, we attempted to apply the 2D V-FEM for the real EC-CHF structure to compute all the parameters in the loss formula (7) except for the core and cladding indices, which are the total power $P_{g}$, the cladding field amplitude coefficient $A$, and the effective index $n_{\text {eff }}$, and the computed results are shown by the thick solid curves in Fig. 5. The core and cladding indices $n_{1}$ and $n_{2}$ remain being approximated by the FSM indices in these regions. In the results, we observed no significant discrepancy depending on equivalent core radius and the results lie between those obtained by the formula approach based on the equivalent SI fiber for two equivalent core radii of $m \Lambda$ and $(m+0.5) \Lambda$. On the other hand, the thick dashed curve represents the result in which the real structure is considered only for evaluating

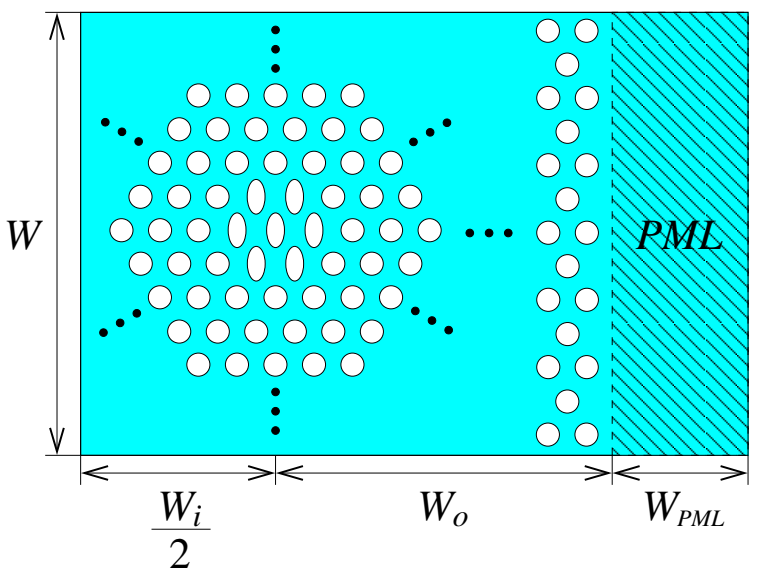

Fig. 6. Computational domain used in directly numerical approaches.

the power and the amplitude coefficient in the loss formula (7). The results are similar to those obtained by the formula approach based on the equivalent SI fiber (thin solid curves).

Finally, to verify the approximate approach based on the loss formula, we apply two directly numerical approaches, which are the complex eigenmode analysis using the 2D V-FEM and the 3D vector finite-element BPM (V-FEBPM) analysis, to real EC-CHF structures including a cladding with a finite lattice structure. Here, the computational domain is shown in Fig. 6 and the cladding region consisting of an air-hole lattice is terminated by a perfectly matched layer (PML) [32]-[34]. In our simulations, $W$ and $W_{P M L}$ are assumed to be $40 \mu \mathrm{m}$ and $2 \mu \mathrm{m}$, respectively. The bending losses, which are computed by the $2 \mathrm{D}$ complex eigenmode analysis using a cylindrical coordinate system, for the cladding region widths of 20.0 to $66.0 \mu \mathrm{m}$ with $2.0 \mu \mathrm{m}$ steps are shown in Fig. 7. In the cladding region with a small width, since confinement loss occurs due to a finite cladding, the computed loss approaches the confinement loss with increasing bending radius. In a large $W_{o}$, on the other hand, the loss decreases with expanding the cladding region, but its abrupt increase is observed for small values of $R / W_{o}$. This is ascribable to a coupling between the guided mode and a cladding mode. Similar peaks in the bending loss curves have also been observed in [24], [35]. To accurately evaluate the bending loss, the cladding region width is required to be large enough (under without such undesirable coupling), and thus lower bounds for the computed losses as a function of the cladding width $W_{o}$ shown in Fig. 7 will provide the net bending loss at a bending radius. In Fig. 5, the open circles and the pluses denote the bending losses finally obtained by the 2D complex eigenmode analyses using a cylindrical coordinate system and the equivalent straight structure model, respectively, and the squares are by the $3 \mathrm{D}$ V-FEBPM. In the 3D V-FEBPM simulation, a cylindrical coordinate system is adopted. There is excellent agreement among these directly numerical approaches, which are two approaches based on the 2D complex eigenmode analysis and the 3D V-FEBPM approach. The result obtained by the formula approach based on the equivalent SI fiber for $a=(m+0.5) \Lambda$ (thin solid curve) is in good agreement with the directly numerical approaches for the real bent EC-CHF 


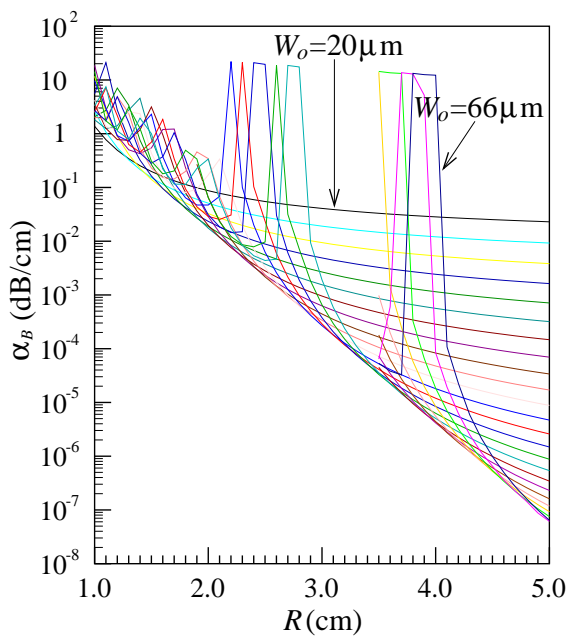

Fig. 7. Dependence of the computational domain on the bending loss computed by the $2 \mathrm{D}$ complex eigenmode analysis.

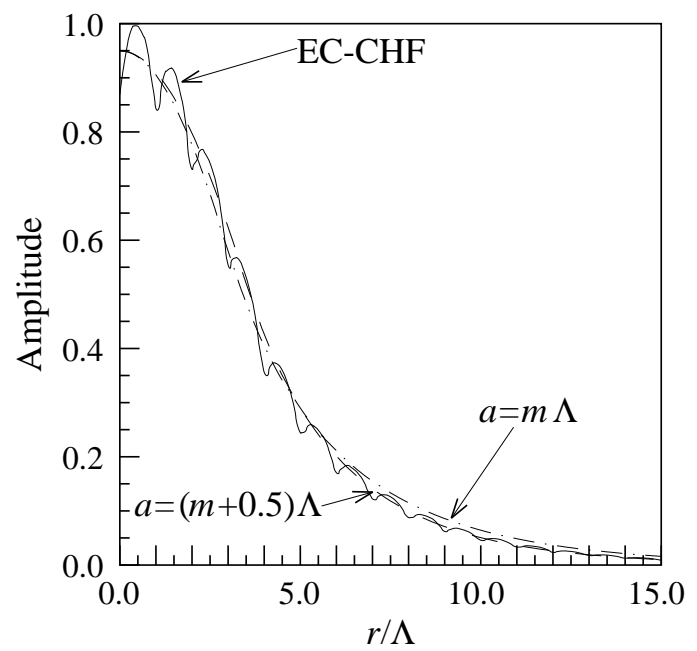

Fig. 8. Field distributions on the $x$ axis of a three-ring $y$ EC-CHF (solid curve) and equivalent SI fibers with $a=(m+0.5) \Lambda$ (dashed curve) and $m \Lambda$ (dashed-dotted curve).

structure. Figure 8 shows mode field distributions on the $x$ axis and the solid curve corresponds to the real EC-CHF structure, while the dashed and dashed-dotted curves correspond to the equivalent SI fibers with $a=(m+0.5) \Lambda$ and $m \Lambda$, respectively. We can see that the agreement between the EC-CHF (solid curve) and the equivalent SI fiber with $a=(m+0.5) \Lambda$ is fairly good, especially in the cladding region. This explains that the equivalent SI fiber with $a=(m+0.5) \Lambda$ more accurately approximates the real structure than that with $a=m \Lambda$, and thus we may be able to have an approximate equivalent core radius by comparing the mode field of equivalent SI fibers with that of the EC-CHF. Figure 9 shows the dependence of bending radius on the equivalent core radius, which satisfies that the bending loss obtained by the standard loss formula (3) with it coincides with the 2D V-FEM calculation using a cylindrical coordinate system. It is remarkable that the variation of the effective core radius with bending radius abruptly becomes smaller, for a bending radius larger than approximately 2.0 $\mathrm{cm}$. Thus, for a bending radius exceeding a certain threshold
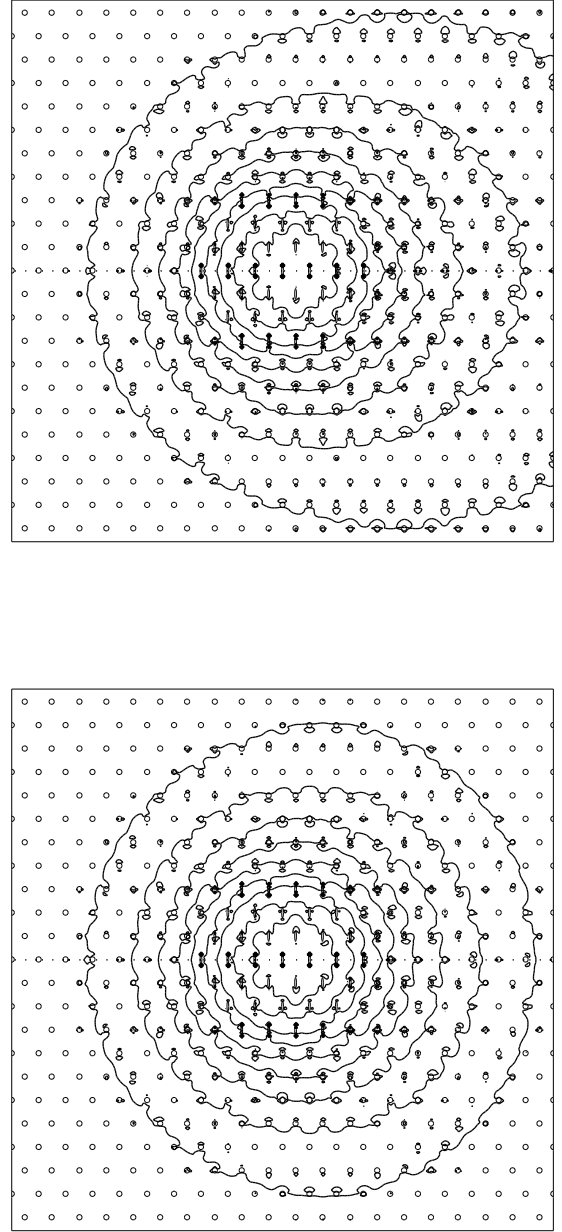

Fig. 9. Field distribution in a bent three-ring $y \mathrm{EC}-\mathrm{CHF}$ with $R=$ (a) $1 \mathrm{~cm}$ and (b) $2 \mathrm{~cm}$.

value, the dependence of equivalent core radius on the formula approach may be relatively small. However, in fact, the definition of equivalent core radius depends on many physical parameters, such as wavelength, air-hole sizes, lattice pitch, and the number of air-hole ring in the core lattice. Perhaps, light confinement in the core region will be the most important factor. Thus, it is not easy to derive a widely available relation to define the equivalent core radius in the standard bending loss formular for EC-CHFs and the detailed investigation will be the subject in the future. The standard formula derived for round fibers having an axially symmetric profile is effective for accurately predicting the bending loss of EC-CHFs, if the equivalent core radius is suitably defined.

Figures 9(a) and 9(b) display the field distributions in the bent three-ring $y \mathrm{EC}-\mathrm{CHFs}$ with bending radii of 1.0 and $2.0 \mathrm{~cm}$, respectively, and are computed by the $2 \mathrm{D}$ complex eigenmode analysis using a cylindrical coordinate system. A significant radiation field caused by the bend is observed in the bending radius of $1.0 \mathrm{~cm}$. On the other hand, the bent fiber with $R=2.0 \mathrm{~cm}$ preserves the mode field in the straight condition approximately. It seems that the accuracy of bending losses (thin solid curve in Fig. 5) evaluated by the standard formula is degraded for the field deformation caused by a small 


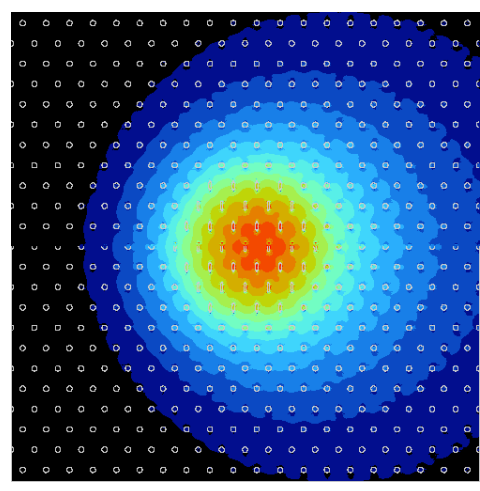

(a)

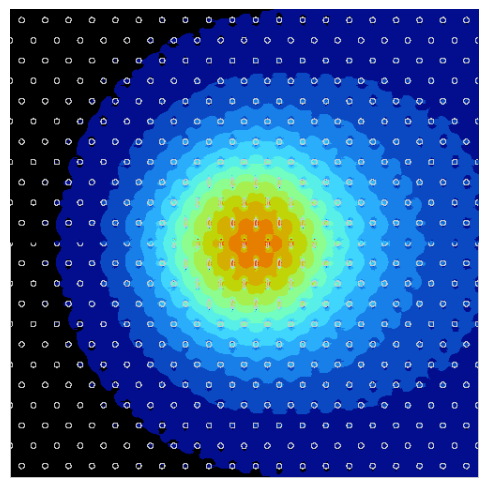

(d)

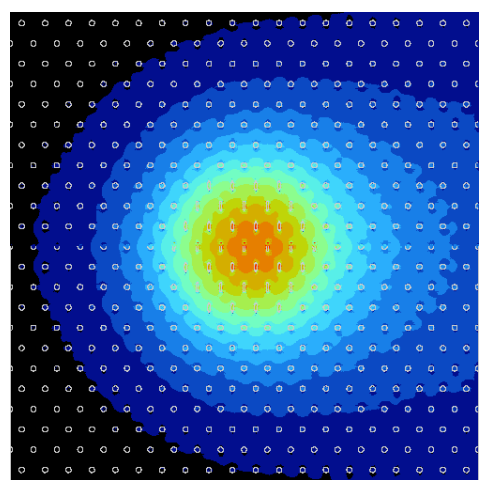

(b)

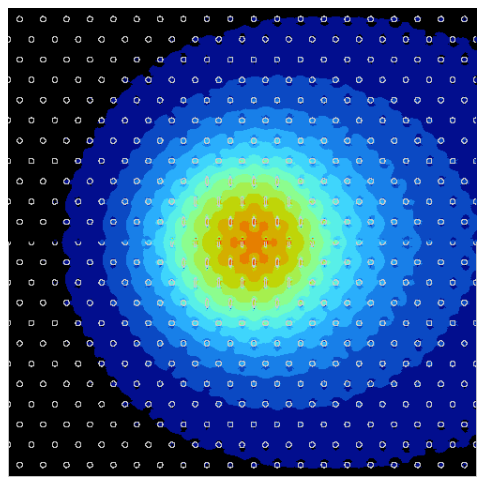

(e)

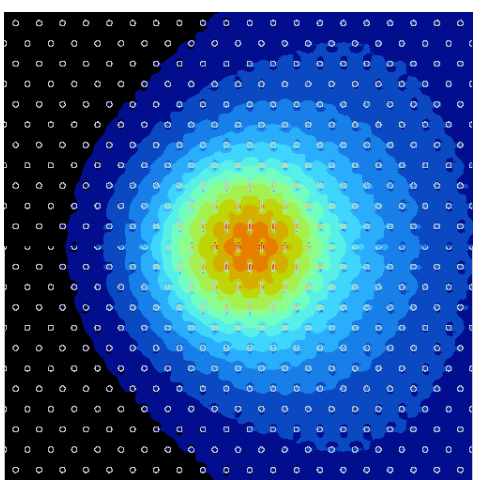

(c)

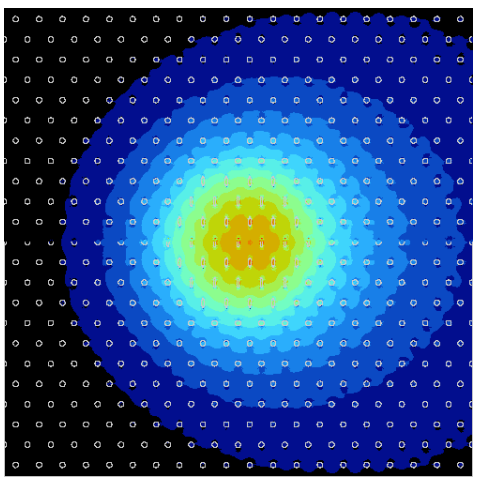

(f)

Fig. 10. Mode propagation along a bent three-ring $y$ EC-CHF with a bending radius $1 \mathrm{~cm}$ over a length of $5 \mathrm{~mm}$. (a) $z=0.25 \mathrm{~mm}$. (b) $z=0.5 \mathrm{~mm}$. (c) $z=0.75 \mathrm{~mm}$. (d) $z=1 \mathrm{~mm}$. (e) $z=3 \mathrm{~mm}$. (f) $z=5 \mathrm{~mm}$.

bend. Moreover, we demonstrate the mode propagation along a bent three-ring $y \mathrm{EC}-\mathrm{CHF}$ with a bending radius $1.0 \mathrm{~cm}$ over a length of $5.0 \mathrm{~mm}$ by using the 3D V-FEBPM simulation in Fig. 10. We will see that, as the mode propagates down the bent fiber, its power radiates into the cladding region.

\section{NUMERICAL EXAMPLES}

To verify the above discussion, we have applied a few approaches to EC-CHFs having other structures. Figures 11, 12, and 13 show the bending losses of a three-ring $x \mathrm{EC}$-, four-ring $y \mathrm{EC}-$, and four-ring $x \mathrm{EC}-\mathrm{CHFs}$, respectively. The open circles denote the results for the real EC-CHF structures obtained by the $2 \mathrm{D}$ complex eigenmode analysis using a cylindrical coordinate system. The thin solid and thick dashed curves correspond to the results obtained by the formula approach using (3) based on the equivalent SI fiber, and the formula approach using (7) in which the 2D V-FEM is applied only to evaluate the power and amplitude coefficient for the real structure, respectively. The bending loss of EC-CHF seems to be hardly influenced by the direction of elliptical holes in the core lattice. This is because the EC-CHFs evaluated here are approximately weakly guiding, as seen from Fig. 4. The formula approach based on the equivalent SI fiber will provide a more accurate result for a lower relative FSM index difference given by $\Delta$, and here $\Delta=0.3717$ and $0.3722 \%$ for the $y \mathrm{EC}$ - and $x \mathrm{EC}$-CHFs, respectively, at $\lambda=1.55 \mu \mathrm{m}$.
In addition, we observed good agreement between the loss curves for the real EC-CHFs and the equivalent SI fibers with $a=(m+0.5) \Lambda$ through the results shown in Figs. 5, 11, 12, and 13. This means that EC-CHFs possess bending properties comparable to those of standard weakly guiding fibers.

\section{CONCLUSION}

Various approaches have been applied and compared for the bending loss evaluations of single-polarization EC-CHFs, which have an elliptical-hole lattice structure in the core and, thus, possess more peculiar transmission properties compared with conventional HFs. In using the loss formula for an equivalent SI fiber, the definition of the equivalent core radius was found to strongly affect the accuracy of bending loss evaluations. However, when an EC-CHF is approximately weakly guiding (i.e. has a low relative FSM index difference), the result obtained by the formula approach based on the equivalent SI fiber will be in good agreement with directly numerical approaches for the real structure including a cladding with a finite lattice structure. As a result, we can conclude that, under a suitable definition of an equivalent core radius, the standard loss formula derived for standard weakly guiding SI fibers is strongly effective for predicting the bending losses of general HFs. 


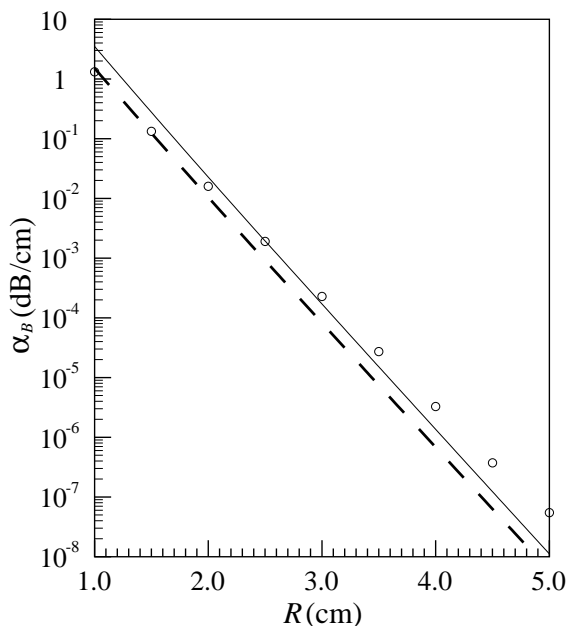

Fig. 11. Bending loss property of a three-ring $x$ EC-CHF. Circles, the 2D complex eigenmode analysis. Thin solid curve, the formula approach using (3) based on the equivalent SI fiber. Thick dashed curve, the formula approach using (7) in which the real EC-CHF structure is considered only for evaluating $P_{g}$ and $A$.

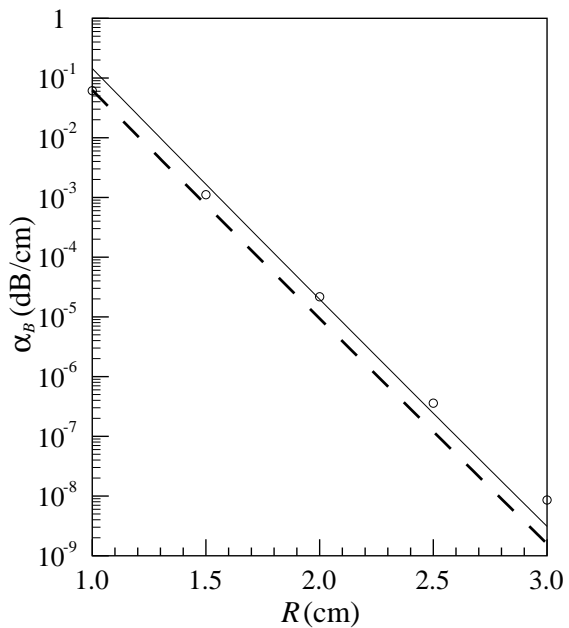

Fig. 12. Bending loss property of a four-ring $y \mathrm{EC}-\mathrm{CHF}$.

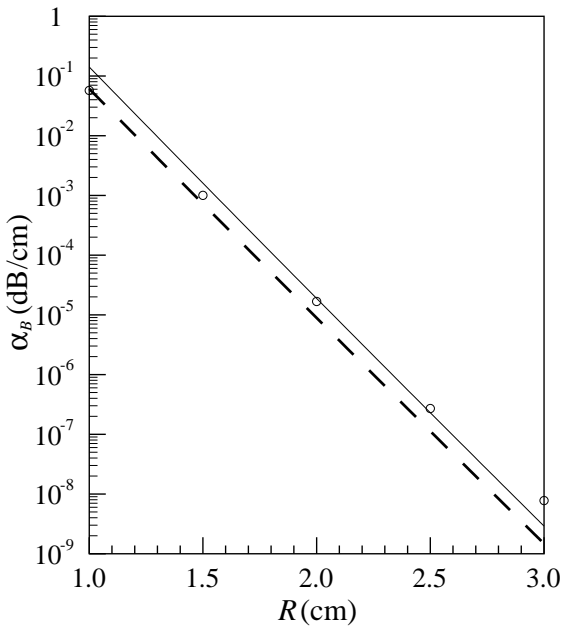

Fig. 13. Bending loss property of a four-ring $x$ EC-CHF.

\section{REFERENCES}

[1] D.N. Payne, A.J. Barlow, and J.J.R. Hansen, "Development of low- and high-birefringence optical fibers ," IEEE J. Quantum Electron., QE-18, pp.477-488, 1982

[2] J. Noda, K. Okamoto, and Y. Sasaki, "Polarization-maintaining fibers and their applications ," IEEE/OSA J. Lightwave Technol., LT-4, pp.1071-1089, 1986.

[3] M.J. Messerly, J.R. Onstott, and R.C. Mikkelson, "A broad-band single polarization optical fiber ", IEEE/OSA J. Lightwave Technol., 9, pp.817820, 1991

[4] J.C. Knight, T.A. Birks, P.St.J. Russell, and D.M. Atkin, "All-silica singlemode optical fiber with photonic crystal cladding ," OSA Opt. Lett., 21, pp.1547-1549, 1996.

[5] J.C. Knight, T.A. Birks, P.St.J. Russell, and D.M. Atkin, "All-silica singlemode optical fiber with photonic crystal cladding : errata " OSA Opt. Lett., 22, pp.484-485, 1997.

[6] P.St.J. Russell, "Photonic-crystal fibers ," IEEE/OSA J. Lightwave Technol., 24, pp.4729-4749, 2006.

[7] M. Eguchi and Y. Tsuji, "Squeezed lattice elliptical-hole holey fiber with extremely high birefringence ," OSA Opt. Lett., 33, pp.1792-1794, 2008.

[8] H. Kubota, S. Kawanishi, S. Koyanagi, M. Tanaka, and S. Yamaguchi, "Absolutely single polarization photonic crystal fiber ," IEEE Photon. Technol. Lett., 16, pp.182-184, 2004.

[9] J.R. Folkenberg, M.D. Nielsen, and C. Jakobsen, "Broadband singlepolarization photonic crystal fiber " OSA Opt. Lett., 30, pp.1446-1448, 2005.

[10] T. Schreiber, F. Röser, O. Schmidt, J. Limpert, R. Iliew, F. Lederer, A. Petersson, C. Jacobsen, K.P. Hansen, J. Broeng, and A. Tünnermann, "Stress-induced single-polarization single-transverse mode photonic crystal fiber with low nonlinearity ," OSA Opt. Express, 13, pp.7621-7630, 2005.

[11] T.A. Birks, J.C. Knight, and P.St.J. Russell, "Endlessly single-mode photonic crystal fiber ," OSA Opt. Lett., 22, pp.961-963, 1997.

[12] M. Eguchi and Y. Tsuji, "Single-mode single-polarization holey fiber using anisotropic fundamental space-filling mode ," OSA Opt. Lett., 32, pp.2112-2114, 2007.

[13] M. Eguchi and Y. Tsuji, "Design of single-polarization elliptical-hole core circular-hole holey fibers with zero dispersion at $1.55 \mu \mathrm{m}$," OSA J. Opt. Soc. Am. B, 25, pp.1690-1701, 2008.

[14] E.A.J. Marcatili, "Bends in optical dielectric guides ," Bell Syst. Tech. J., 48, pp.2103-2132, 1969.

[15] D. Marcuse, "Bending losses of the asymmetric slab waveguide ," Bell Syst. Tech. J., 50, pp.2551-2132, 1971.

[16] D. Marcuse, Light Transmission Optics . Van Nostrand, Reinhold, New York, 1972.

[17] D. Marcuse, "Curvature loss formula for optical fibers ", OSA J. Opt. Soc. Am., 66, pp.216-220, 1976.

[18] D. Marcuse, "Bend loss of slab and fiber modes computed with diffraction theory ," IEEE J. Quantum Electron., 29, pp.2957-2961, 1993.

[19] J. Sakai and T. Kimura, "Bending loss of propagation modes in arbitraryindex profile optical fibers ," OSA Appl. Opt., 17, pp.1499-1506, 1978.

[20] D. Marcuse, "Influence of curvature on the losses of doubly clad fibers ," OSA Appl. Opt., 21, pp.4208-4213, 1982.

[21] M. Heiblum and J.H. Harris, "Analysis of curved optical waveguides by conformal transformation ," IEEE J. Quantum. Electron., QE-11, pp.75-83, 1975.

[22] J.D. Mariani and G. Rodrigue, "Study of optically induced effects due to bending and twisting using the vector finite-element method " OSA $J$. Opt. Soc. Am. B, 23, pp.1743-1751, 2006.

[23] M.D. Nielsen, N.A. Mortensen, M. Albertsen, J.R. Folkenberg, A. Bjarklev, and D. Bonacinni, "Predicting macrobending loss for large-mode area photonic crystal fibers ," OSA Opt. Express, 12, pp.1775-1779, 2004.

[24] J. Olszewski, M. Szpulak, and W. Urbańczyk, "Effect of coupling between fundamental and cladding modes on bending losses in photonic crystal fibers," OSA Opt. Express, 13, pp.6015-6022, 2005.

[25] S. Février, R. Jamier, J.-M. Blondy, S.L. Semjonov, M.E. Likhachev, M.M. Bubnov, E.M. Dianov, V.F. Khopin, M.Y. Salganskii, and A.N. Guryanov, "Low-loss singlemode large mode area all-silica photonic bandgap fiber ," OSA Opt. Express, 14, pp.562-569, 2006.

[26] N.H. Vu, I.-K. Hwang, and Y.-H. Lee, "Bending loss analyses of photonic crystal fibers based on the finite-difference time-domain method ," OSA Opt. Lett., 33, pp.119-121, 2008.

[27] Y. Tsuji and M. Koshiba, "Adaptive mesh generation for full-vectorial guided-mode and beam-propagation solutions ", IEEE J. Select. Topics Quantum Electron., 6, pp.163-169, 2000.

[28] Y. Tsuji and M. Koshiba, "Finite element beam propagation method with perfectly matched layer boundary conditions for three-dimensional optical waveguides ," Int. J. Numer. Model., 13, pp.115-126, 2000.

[29] M. Eguchi, M. Koshiba, and Y. Tsuji, "Dispersion compensation based on dual-mode optical fiber with inhomogeneous profile core ," IEEE/OSA J. Lightwave Technol., 14, pp.2387-2394, 1996. 
[30] M. Eguchi and S. Horinouchi, "Finite-element modal analysis of largecore multimode optical fibers ," OSA Appl. Opt., 43, pp.2163-2167, 2004.

[31] M. Eguchi and S. Horinouchi, "Finite-element modal analysis of largecore multimode optical fibers: erratum ," OSA Appl. Opt., 43, p.3158, 2004.

[32] J.-P. Berenger, "A perfectly matched layer for the absorption of electromagnetic waves ,"J. Comp. Phys., 114, pp.185-200, 1994.

[33] F.L. Teixeira and W.C. Chew, "General closed-form PML constitutive tensors to match arbitrary bianisotropic and dispersive linear media ," IEEE Microwave Guided Wave Lett., 8, pp.223-225, 1998.

[34] Y. Tsuji and M. Koshiba, "Complex modal analysis of curved optical waveguides using a full-vectorial finite element method with perfectly matched layer boundary conditions ," Electromagnetics J., 24, pp.39-48, 2004.

[35] T. Martynkien, J. Olszewski, M. Szpulak, G. Golojuch, W. Urbanczyk, T. Nasilowski, F. Berghmans, and H. Thienpont "Experimental investigations of bending loss oscillations in large mode area photonic crystal fibers ," OSA Opt. Express, 15, pp.13547-13556, 2007.

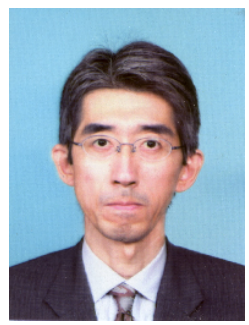

Masashi Eguchi (M'93) was born in Sapporo, Japan, on August 9, 1962. He received the B.S degree in electronic engineering from Kitami Institute of Technology, Kitami, in 1985 and the M.S. and Ph.D degrees in electronic engineering from Hokkaido University, Sapporo, Japan, in 1987, 1991, respectively.

He joined Sony Co., Ltd., in 1987. From 1991 to 1995 , he was with the Department of Industrial Design, Sapporo School of the Arts, Sapporo, Japan. And from 1995 to 1998, he was an Associate Professor of Center for Multimedia Aided Education at Muroran Institute of Technology, Muroran, Japan. Since 1998, he has been an Associate Professor of the Faculty of Photonics Science, Chitose Institute of Science and Technology, Chitose, Japan. He has been engaged in research on various optical fibers, dielectric waveguides, optical solitons, and applications of finite element method and other electromagnetic wave analysis methods.

Dr.Eguchi is a member of the Optical Society of America(OSA), the Institute of Electronics, Information and Communication Engineers(IEICE), and the Institute of Electrical and Electronic Engineers (IEEE).

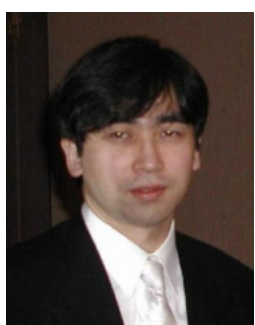

Yasuhide Tsuji (M'97) was born in Takikawa, Japan, on December 31, 1967. He received the B.S. M.S., and Ph.D. degrees in electronic engineering from Hokkaido University, Sapporo, Japan, in 1991, 1993, and 1996, respectively.

In 1996, he joined the Department of Applied Electronic Engineering, Hokkaido Institute of Technology, Sapporo, Japan. From 1997 to 2004, he was an Associate Professor of Electronics and Information Engineering at Hokkaido University. Since 2004, he has been an Associate Professor of Electrical and Electronic Engineering at Kitami Institute of Technology, Kitami, Japan. He has been engaged in research on optical wave electronics.

Dr. Tsuji is a member of the Institute of Electronics, Information and Communication Engineers (IEICE), the Japan Society of Applied Physics, the Optical Society of America (OSA), and IEEE. In 1997 and 1999, he was awarded the Excellent Paper Award from IEICE. In 2000, he has received the Third Millennium Medal from IEEE.

\section{FIGURE CAPTIONS}

Fig. 1. Elliptical-hole core circular-hole HF. (a) The $y$ EC-CHF. (b) The $x$ EC-CHF.

Fig. 2. Coordinate system for the standard loss formula (3).

Fig. 3. Coordinate system for the formula (9) based on diffraction theory.

Fig. 4. Dispersion property for the guided mode of a singlepolarization three-ring $y$ EC-CHF (solid curve) and equivalent SI fibers with $a=3.5 \Lambda$ (open circles) and $a=3 \Lambda$ (open squares).

Fig. 5. Bending loss property of a three-ring $y$ EC-CHF. Thin solid curves, the formula approach using (3) based on the equivalent SI fiber. Thin dashed curves, the formula approach using (7) based on the equivalent SI fiber with the approximate scalar FEM. Thin dashed-dotted curves, the formula approach using (9) based on the equivalent SI fiber with the approximate scalar FEM. Thick solid curves, the formula approach using (7) based on the equivalent SI fiber in which the real ECCHF structure is considered for evaluating all the parameters in (7) ( $P_{g}, A$, and $\left.n_{\text {eff }}\right)$. Thick dashed curves, the formula approach using (7) based on the equivalent SI fiber in which the real EC-CHF structure is considered only for evaluating $P_{g}$ and $A$. Open circles and pluses, the 2D complex eigenmode analyses using a cylindrical coordinate system and the equivalent straight structure model, respectively, for the real EC-CHF structure. Squares, the 3D V-FEBPM for the real ECCHF structure.

Fig. 6. Computational domain used in directly numerical approaches.

Fig. 7. Dependence of the computational domain on the bending loss computed by the 2D complex eigenmode analysis.

Fig. 8. Field distributions on the $x$ axis of a three-ring $y \mathrm{EC}$ CHF (solid curve) and equivalent SI fibers with $a=(m+$ $0.5) \Lambda$ (dashed curve) and $m \Lambda$ (dashed-dotted curve).

Fig. 9. Field distribution in a bent three-ring $y \mathrm{EC}-\mathrm{CHF}$ with $R=$ (a) $1 \mathrm{~cm}$ and (b) $2 \mathrm{~cm}$.

Fig. 10. Mode propagation along a bent three-ring $y \mathrm{EC}$-CHF with a bending radius $1 \mathrm{~cm}$ over a length of $5 \mathrm{~mm}$. (a) $z=$ $0.25 \mathrm{~mm}$. (b) $z=0.5 \mathrm{~mm}$. (c) $z=0.75 \mathrm{~mm}$. (d) $z=1 \mathrm{~mm}$. (e) $z=3 \mathrm{~mm}$. (f) $z=5 \mathrm{~mm}$.

Fig. 11. Bending loss property of a three-ring $x \mathrm{EC}-\mathrm{CHF}$. Circles, the 2D complex eigenmode analysis. Thin solid curve, the formula approach using (3) based on the equivalent SI fiber. Thick dashed curve, the formula approach using (7) in which the real EC-CHF structure is considered only for evaluating $P_{g}$ and $A$.

Fig. 12. Bending loss property of a four-ring $y \mathrm{EC}-\mathrm{CHF}$.

Fig. 13. Bending loss property of a four-ring $x \mathrm{EC}-\mathrm{CHF}$. 